\title{
Memory after treatment for acute lymphoblastic leukaemia
}

\author{
J Rodgers, P G Britton, R G Morris, J Kernahan, A W Craft
}

\begin{abstract}
Long term survivors of acute lymphoblastic leukaemia (ALL) often experience cognitive difficulties, which may be related to impairment of memory function. Memory ability has been studied in a group of survivors of ALL along with sibling controls and in children who have received treatment for other forms of cancer. Children in the ALL group were found to have significant deficits in memory function in tasks which required the application of strategic planning behaviour. These deficits are potentially remediable by educational strategies.
\end{abstract}

A majority of children now survive acute lymphoblastic leukaemia (ALL) but there has been an increasing awareness in recent years of the resultant intellectual deficits. ${ }^{12}$ The assumption has been that cranial irradiation has been responsible for the decline in intellect and attempts have been made to replace it with systemic and intrathecal chemotherapy. There appears to be a global fall in intelligence quotient (IQ) in many children that may be largely due to deficits in memory function. ${ }^{3}$ However, the details of where within the human memory system these deficits occur has not been previously identified. Normal memory function in both children and adults requires both active organisation and structuring of incoming material as well as the passive storage of information. ${ }^{4}$

A series of studies in Newcastle upon Tyne 56 has shown intellectual deficts in childhood ALL survivors when compared with both sibling controls and those treated for solid tumours when assessed using the British ability scales (BAS). The deficits seem to persist with time and are no fewer in patients who have been given $18 \mathrm{~Gy}$ cranial irradiation than in those given $24 \mathrm{~Gy}^{7}$ The shortfall seen in the ALL patients on the subtests of the BAS for 'speed of

Table 1 Age at diagnosis and at assessment of leukaemia and tumour patients and their sibling controls

\begin{tabular}{|c|c|c|c|c|c|}
\hline \multirow{2}{*}{$\begin{array}{l}\text { Subject } \\
\text { group }\end{array}$} & \multirow{2}{*}{$\begin{array}{l}\text { No of } \\
\text { subjects }\end{array}$} & \multicolumn{2}{|c|}{ Age at diagnosis (months) } & \multicolumn{2}{|c|}{ Age at assessment (months } \\
\hline & & Range & Mean $(S D)$ & Range & Mean (SD) \\
\hline \multicolumn{6}{|l|}{$\begin{array}{l}\text { ALL: } \\
18 \mathrm{~Gy}\end{array}$} \\
\hline Patients & 50 & $10-162$ & $55 \cdot 5(36 \cdot 6)$ & $55-253$ & $124(41 \cdot 1)$ \\
\hline Siblings & 41 & - & - & $48-219$ & $112(49 \cdot 8)$ \\
\hline \multicolumn{6}{|l|}{$24 \mathrm{~Gy}$} \\
\hline Patients & 14 & $15-119$ & $53.7(36 \cdot 8)$ & $133-219$ & $210(42 \cdot 9)$ \\
\hline Siblings & 11 & - & - & $112-291$ & $202(58 \cdot 8)$ \\
\hline \multicolumn{6}{|l|}{ Solid tumour: } \\
\hline Patients & 19 & $1-195$ & $95 \cdot 8(63 \cdot 1)$ & $51-264$ & $134(71 \cdot 7)$ \\
\hline Siblings & 13 & - & - & $61-270$ & $151(63 \cdot 0)$ \\
\hline
\end{tabular}

information processing and matrices' could suggest a deficit in the ability to formulate strategies or plans of action within the memory system. To clarify further the reasons for intellectual impairment a detailed assessment of memory function has been undertaken.

\section{Subjects and methods}

Sixty four children in long term remission from ALL with 52 sibling controls, and 19 survivors of solid tumours with 13 siblings were studied. Of the children with ALL, 50 had received 18 Gy cranial irradiation and 14 had received 24 Gy. Further details are given in table 1. Each child was given a series of tests to determine intellectual and memory function. The BAS subtests appropriate to the child's age were administered and results of these have been given elsewhere. ${ }^{7}$ In addition each received the following:

(1) The Cambridge automated neuropsychological test battery (CANTAB). ${ }^{8}$ This is a set of computerised memory assessment procedures using a touch sensitive screen (Microvitec Touch Tech 501) on a BBC microcomputer. The tests selected were (a) the reaction time test (RTT), a test of choice reaction time; (b) delayed matching to sample test (DMTS), a measure of immediate memory for visual information; and (c) the delayed response test (DREP), which tests short term memory of material presented in a complex visual array.

(2) Immediate and delayed free recall test (IDFR). Four lists of 10 nouns were presented on a cassette recording at two speeds of presentation-that is, at one and two seconds per word. Two of the lists were presented at the beginning of the testing session and two at the end. The number of words correctly recalled immediately after presentation of each list was recorded (the immediate condition). After recalling both lists at each presentation, subjects were required to recall the first list again without a further chance to hear it (the delayed condition).

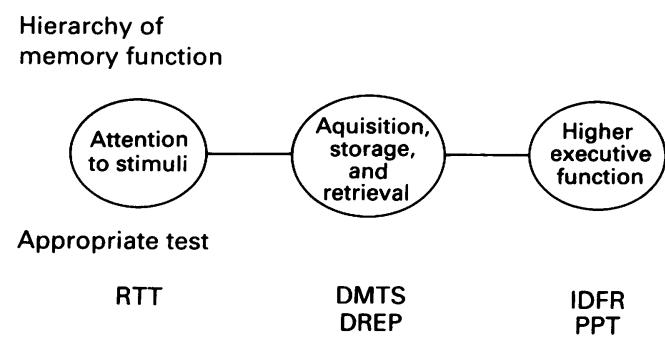

Figure 1 The memory process, showing the appropriate test for each memory function. 
(3) Picture probe task (PPT). ${ }^{y}$ Six cards were presented face down in a row and the subject was asked to turn over each in turn, then replace it face down. A seventh card was then turned over and the subject was asked to select one of the original six cards which displayed the same household object. Differences in the


Figure 2. Mean interitem study times at each card position for patients and siblings in the leukaemia groups $(A) 18 G y$ and $(B) 24 G y$ and in the solid tumour group $(C)$ during the picture probe task. number of cards correctly recalled may result from variations in the subjects' use of mnemonic devices such as a rehearsal strategy to aid recall. This possibility was investigated by calculating the mean intertime study times for each group of patients or siblings. This is the interval (in seconds) between the subject replacing one card and picking up the next. This task, unlike the other memory tasks, is self paced: the subject is in control of the rate of the presentation of the 'to be remembered' objects. This makes it possible to determine whether a rehearsal strategy is being employed. If the subject is using a cumulative rehearsal fast finish strategy, which would be the most appropriate mnemonic device for this task, then this can be seen when the interitem study times are plotted graphically. ${ }^{10}$

Figure 1 indicates which areas of the memory process each of the tasks is targeting. The median and mean scores for each test were calculated and comparisons were made between the different subject and sibling groups. The statistical test used was the analysis of variance, with age acting as a covariant where appropriate.

\section{Results}

The major findings for each of the tests are shown in table 2. For RTT, DMTS, and DREP there were no significant differences between subject groups and sibling controls. Similarly, when immediate recall in the IDFR test was examined no significant differences were found at either presentation speed. However, when the number of items correctly recalled during the delayed test in the IDFR was examined, the leukaemia patients in both the $24 \mathrm{~Gy}$ and $18 \mathrm{~Gy}$ groups were found to recall significantly fewer items than sibling controls (24 Gy, $F=6$, $\mathrm{p}=0.01 ; 18 \mathrm{~Gy}, \mathrm{~F}=4.6 \mathrm{p}=0.05$ ). There was no such difference in delayed recall in the group with solid tumours.

In the PPT an analysis of covariance showed that both of the leukaemia groups were found to recall significantly fewer items than their sibling controls $(24 \mathrm{~Gy}, \mathrm{~F}=1 \cdot 8, \mathrm{p}=0.03 ; 18 \mathrm{~Gy}, \mathrm{~F}=5 \cdot 0$, $\mathbf{p}=0.01)$. No such difference was found in the solid tumour group. The interitem study time curves are shown in fig 2 . The solid tumour

Table 2 Mean (SD) results for the tests on all groups, showing the reaction time, and the number or percentage of correct recalls

\begin{tabular}{|c|c|c|c|c|c|c|}
\hline \multirow[t]{2}{*}{ Tests } & \multicolumn{2}{|c|}{$A L L$ at $18 G y$} & \multicolumn{2}{|c|}{$A L L$ at $24 G y$} & \multicolumn{2}{|l|}{ Solid tumour } \\
\hline & Patients & Siblings & Patients & Siblings & Patients & Siblings \\
\hline $\begin{array}{l}\text { Attention: } \\
\text { RTT (seconds) } \\
\text { Basic processes: }\end{array}$ & $70 \cdot 7(85 \cdot 3)$ & $70 \cdot 6(85 \cdot 3)$ & $32 \cdot 3(4 \cdot 5)$ & $32 \cdot 5(7 \cdot 6)$ & $44 \cdot 8(18 \cdot 1)$ & $38 \cdot 2(13 \cdot 5)$ \\
\hline $\begin{array}{l}\text { DMTS (No) } \\
\text { Simultaneous } \\
0 \\
4 \\
12 \\
\text { DREP (\% at level } 8)\end{array}$ & $\begin{array}{l}9.0(1 \cdot 3) \\
7.3(1 \cdot 8) \\
6.5(1.9) \\
6.0(2 \cdot 4) \\
84\end{array}$ & $\begin{array}{l}9 \cdot 0(1 \cdot 4) \\
7 \cdot 4(2 \cdot 0) \\
6.9(2 \cdot 1) \\
6 \cdot 7(2 \cdot 2) \\
87\end{array}$ & $\begin{array}{l}9 \cdot 9(0 \cdot 3) \\
8 \cdot 7(1 \cdot 1) \\
8 \cdot 4(1 \cdot 2) \\
8 \cdot 5(1 \cdot 5) \\
92\end{array}$ & $\begin{array}{l}9 \cdot 7(0 \cdot 4) \\
9 \cdot 1(1 \cdot 2) \\
8 \cdot 5(1 \cdot 7) \\
8 \cdot 7(1 \cdot 5) \\
90\end{array}$ & $\begin{array}{l}9 \cdot 1(1 \cdot 4) \\
7 \cdot 7(1 \cdot 9) \\
7 \cdot 3(2 \cdot 2) \\
5.9(2 \cdot 8) \\
89\end{array}$ & $\begin{array}{l}9 \cdot 0(0 \cdot 9) \\
7 \cdot 3(2 \cdot 2) \\
6 \cdot 3(2 \cdot 7) \\
7 \cdot 1(2 \cdot 7) \\
100\end{array}$ \\
\hline $\begin{array}{l}\text { Executive function: } \\
\text { IDFR (\%): immediate at } \\
1 \text { second } \\
2 \text { seconds } \\
\text { Delayed } \\
\text { PPT (no) }\end{array}$ & $\begin{array}{l}49 \\
50 \\
22 \\
10 \cdot 7(2 \cdot 7)^{*}\end{array}$ & $\begin{array}{l}52 \\
59 \\
27 \\
11 \cdot 9(2 \cdot 8)\end{array}$ & $\begin{array}{l}56 \\
58 \\
20 \\
11 \cdot 2(2 \cdot 4)^{3}\end{array}$ & $\begin{array}{l}61 \\
66 \\
36 \\
13 \cdot 4(1 \cdot 8)\end{array}$ & $\begin{array}{l}42 \\
46 \\
22 \\
10 \cdot 9(4 \cdot 0)\end{array}$ & $\begin{array}{l}46 \\
51 \\
26 \\
13 \cdot 1(3 \cdot 4)\end{array}$ \\
\hline
\end{tabular}

RTT reaction time test; DMTS, delayed matching sample test; DREP, delayed response test; IDFR, immediate and delayed free recal test; PPT, picture probe task.

*Indicating a significant difference in performance. 
patients and all three sibling groups displayed the normal expected pattern if a cumulative rehearsal fast finish strategy is being employed. ${ }^{10}$ However, both leukaemia groups show curves which suggests that no such strategy was being used. This is particularly evident at card 3 where those using a cumulative rehearsal strategy display an increase in time as the number of items to repeat increases to three, whereas the converse is true for the two leukaemia groups at this position.

\section{Discussion}

The results of the overall IQ as measured by the BAS in this group of patients has shown a clear deficit in both of the leukaemia groups when compared with their siblings but no such defict in patients with solid tumours. ${ }^{7}$ Analysis of the component parts of the BAS suggested that deficits in abstract reasoning and planning might be, at least in part, responsible for the global reduction in IQ. The results shown here disaggregate the component parts of the memory and show that the deficit appears to lie in the most sophisticated part of the memory process-that is, the 'central executive'. The tests show no significant differences between patients and siblings in less sophisticated memory functions.

The results of the CANTAB reaction time test and the immediate conditions of the IDFR test show that attentional ability is not affected. Similarly, storage of material is not a problem as shown by the DREP test and the DMTS where all subjects demonstrated ability comparable with their siblings in spatial memory, list learning, visuospatial learning, pattern discrimination, and retention. Retrieval of information is not significantly lower for the leukaemia patients where the DMTS and DREP show ability to retrieve abstract material even over long delay periods.

The results of the delayed condition of the IDFR and the results of the PPT show a consistent deficit in the ALL groups. Figure 2 shows that at least one reason for this is that the children with ALL were not using a cumulative rehearsal strategy. A rehearsal strategy aids recall by transferring the items to be remembered from a memory buffer store into short term memory. If a rehearsal strategy is not employed, as soon as more information to be remembered is introduced (as in the immediate condition of the IDFR), the items already in the buffer store will be lost by a process known as 'retroactive interference' in which incoming material displaces information within the memory buffer. ${ }^{11}$ Therefore subjects not manipulating items in their memory store will recall fewer items during a delayed condition.

Due to the fact that the two leukaemia groups are of unequal size ( $24 \mathrm{~Gy}, \mathrm{n}=14 ; 18 \mathrm{~Gy}$, $\mathrm{n}=50$ ), and those children in the 24 Gy group are somewhat older and had been diagnosed longer, it would be inadvisable at the present time to assume that these two populations are a homogenous group. It is possible that the lack of differences between these groups may result from factors other than specific components of the irradiation treatment. Nevertheless, the children with ALL do appear to be displaying 'strategic unawareness', in other words the memory deficit present in the ALL population appear to be of metamemorial nature rather than a structural defect. The potential importance of these findings lies in the fact that children with this type of deficit may be amenable to intervention and training. Specific training to improve awareness of rehearsal strategies may well overcome some of the global deficits shown by many children who have survived ALL. Remediation may be attempted through the adoption of an intervention programme. Here, those children not using task appropriate memory strategies expected for their age could be identified during routine clinic visits using a simple battery of memory tasks. These children could then undergo a training programme. The programme would be designed to teach the adoption, maintenance, and generalisation of memory aids. Through this method an awareness of techniques such as labelling, that is, asking a child to name a remembered object, and rehearsal, as already mentioned, could be achieved. Attempts at remediation have been made among other groups exhibiting similar deficits with varying results. $^{12}$

Overall treatment strategies for ALL are changing in an attempt to minimise intellectual damage. However, there are many survivors treated on previous regimens who could potentially be helped to optimum functioning.

J Rogers was supported by the Tyneside Leukaemia Research Association.

1 Said JA, Waters BG, Cousens P, Stevens M. Neuropsychological sequelae of central nervous system prophylaxis in survivors of childhood acute lymphoblastic leukaemia. 7 Consult Clin Psychol 1989;57:251-6.

2 Eiser C. Cognitive deficits in children treated for leukaemia. Arch Dis Child 1991;66:164-8.

3 Brouwers P, Riccardi R, Fedio P, Poplack D. Longterm neuropsychologic sequelae of childhood leukaemia: correlation with CT scan abnormalities. I Pediatr 1985;106: 723-8

4 Baddeley AD. Human memory: theory and practice. Howe: Lawrence and Erlbaum Associates, 1990

5 Twaddle V, Britton PG, Craft AW, Noble TC, Kernahan J. Intellectual function after treatment for leukaemia and solid Intellectual function after treatment for le

6 Twaddle V, Britton PG, Kernahan J, Craft AW. Intellect after malignancy. Arch Dis Child 1986;61:700-2.

7 Rodgers J, Britton PG, Kernahan J, Craft AW. Two doses of cranial irradiation compared in acute lymphoblastic cranial irradiation compared in acute

8 Evenden J, Morris RG, Sahakian BJ, Owen A, Robbins T. The Cambridge automated neuropsychological test battery The Cambridge automated neuropsychological test batten
(CANTAB). Cambridge: Paul Frey Associates, 1991 .

9 Belmont JM, Butterfield EC. Learning strategies as determinants of mental deficiencies. Cognitive Psychology 1971; minants of

10 Butterfield EC, Belmont JM. Assessing and improving the executive cognitive functions of mentally retarded people. In: Bialer I, Sternlicht M, eds. Psychological issues in mental retardation. New York: Psychological Dimensions, 1977

11 Keppel G. Retroactive and proactive inhibition. In: Dixon TR, Horton DL, eds. Verbal behaviour and general behaviour theory. Englewood Cliffs, NJ: Prentice Hall, 1968

12 Kramer JJ, Engle RW. Teaching awareness of strategic behaviour in combination with strategy training: effects of children's memory performance. $\mathcal{f}$ Exp Child Psychol 1981;2:513-30 